\title{
Original
}

\section{Age-related associations between psychological characteristics and pain intensity among Japanese patients with temporomandibular disorder}

\author{
Osamu Komiyama1), Ryoko Obara1), Takashi Iida1), Hitoshi Nishimura1), \\ Masakazu Okubo1), Takashi Uchida1), Michiharu Shimosaka1), Noriyuki Narita1), \\ Hideo Niwa1), Hideyuki Kubo'), Antoon De Laat²), Misao Kawara1), \\ and Yasuhide Makiyama1) \\ 1)Orofacial and Head Pain Clinic, Nihon University Hospital at Matsudo, Matsudo, Japan \\ 2)Department of Oral Health Sciences, KU Leuven and Dentistry, University Hospital Leuven, \\ Leuven, Belgium
}

(Received May 3, 2014; Accepted July 10, 2014)

\begin{abstract}
We investigated the relationship between pain intensity and psychosocial characteristics in patients with temporomandibular disorder (TMD). Participants with painful TMD, according to the Research Diagnostic Criteria for TMD (RDC/TMD), were recruited from our clinic and classified into six age groups: 15 to 24,25 to 34,35 to 44,45 to 54,55 to 64 , and 65 to 85 years (Groups A through $F$, respectively). Self-reported present pain intensity and worst pain intensity during the past 6 months were ascertained using a numeric rating scale (0 to 10). Depression and somatization scores were evaluated using the RDC/ TMD axis II questionnaire. Among women, worst pain intensity was significantly lower in Groups $E$ and $F$ than in Groups B and $C(P<\mathbf{0 . 0 5})$. In Groups $A$, $\mathrm{C}$, and $\mathrm{D}$, depression scores were significantly higher in women than in men $(P<0.05)$. Among women, depression score was significantly lower in Group $F$ than in Groups A through $\mathrm{C}(P<\mathbf{0 . 0 5})$. In Groups A through $D$, somatization scores were significantly higher for women than for men $(P<0.05)$. Depression score and TMD symptom severity appear to decrease with age in women. ( $\mathrm{J}$ Oral Sci 56, 221-225, 2014)
\end{abstract}

Correspondence to Dr. Osamu Komiyama, Orofacial and Head Pain Clinic, Nihon University Hospital at Matsudo, 2-870-1 Sakaecho-nishi, Matsudo, Chiba 271-8587, Japan

Fax:+81-47-360-9615 E-mail: komiyama.osamu@nihon-u.ac.jp doi.org/10.2334/josnusd.56.221

DN/JST.JSTAGE/josnusd/56.221
Keywords: temporomandibular disorder; aging; pain; psychological characteristics.

\section{Introduction}

Signs and symptoms of temporomandibular disorder (TMD) have been reported in all examined populations. The prevalence of TMD is low in children, increases during adolescence and young adulthood, and appears to peak during midlife (1). In older adults, the prevalence of reported TMD symptoms decreases with age; however, data on the development of clinically recorded signs are not conclusive (2). Some studies reported an increase in the prevalence of TMD signs with age $(3,4)$. Thus, confusion remains regarding the prevalence of TMD signs and symptoms among the elderly. Anatomical factors and dental occlusion are now regarded as less important etiological factors for TMD, while psychosocial and general health factors have been increasingly implicated in TMD (5). The many unresolved issues in this field highlight the need for new research.

Epidemiological and clinical studies of TMD confirm that it is fundamentally a pain problem-more specifically, a chronic pain problem (6). Somatization, which may be defined for the present purposes as the reporting of non-pain-related physical symptoms, is associated with depression (7). A study of the association of psychological factors with TMD treatment outcome concluded that outcomes are threatened if somatization issues are 
Table 1 Characteristics of participants by sex, muscle/TMJ pain ratio, and chronic pain status

\begin{tabular}{lccccccc}
\hline Age group (years) & $15-24$ & $25-34$ & $35-44$ & $45-54$ & $55-64$ & $65-84$ & Total \\
\hline Women/men & $151 / 73$ & $215 / 117$ & $166 / 53$ & $127 / 44$ & $205 / 61$ & $155 / 70$ & $1019 / 418$ \\
(total) & $(224)$ & $(332)$ & $(219)$ & $(171)$ & $(266)$ & $(225)$ & $(1437)$ \\
Muscle/TMJ pain & $158 / 66$ & $274 / 58$ & $181 / 38$ & $127 / 44$ & $213 / 53$ & $180 / 45$ & $1133 / 304$ \\
Chronic pain (>6 months) & 86 & 118 & 84 & 61 & 82 & 48 & 479 \\
$(\%)$ & $(38.4 \%)$ & $(35.5 \%)$ & $(38.4 \%)$ & $(35.7 \%)$ & $(30.8 \%)$ & $(21.3 \%)$ & $(33.3 \%)$ \\
\hline
\end{tabular}

not addressed in TMD patients $(6,7)$.

We therefore conducted a cross-sectional study of pain intensity and psychological characteristics, such as depression and somatization, among university hospitalbased TMD patients classified by age group, as pain intensity and psychosocial characteristics may vary by age group.

\section{Participants}

\section{Methods}

Participants were recruited from among patients in our clinic who sought treatment for orofacial pain during the period from 2007 through 2010 (total, 3,645 patients). Questionnaires were given to all patients with painful TMD, according to the Research Diagnostic Criteria for Temporomandibular Disorders (RDC/TMD) $(1,437$ patients), including myofascial pain (Ia or Ib), arthralgia (IIIa), and osteoarthritis of the temporomandibular joint (TMJ) (IIIb) (8). Exclusion criteria were metabolic disease (e.g., diabetes, hyperthyroidism), neurological disorders (e.g., dyskinesia, trigeminal neuralgia), vascular disease (e.g., migraine, hypertension), neoplasia, history of drug abuse, recent facial or cervical trauma (e.g., whiplash), current use of medications, and receipt of other treatments.

For the purpose of comparison, the patients were classified into six age groups: 15 to 24,25 to 34,35 to 44,45 to 54,55 to 64 , and 65 to 85 years (Groups A through F, respectively), as in a previous study $(9,10)$.

All participants were informed of the study goals and design in the description of the questionnaire. The local ethics committee approved the study (EC11-014), which adhered to the guidelines of the Helsinki Declaration.

\section{Self-reported measures}

Illness duration (defined as time since onset of painful TMD) was classified as acute ( $<6$ months) or chronic $(>6$ months). Present pain intensity and worst pain intensity during the past 6 months were reported using the same 0 through 10 numeric rating scale (NRS) (8). As for psychosocial characteristics, depression and somatization scores were evaluated using the RDC/TMD axis II questionnaire (8).

\section{Statistical analysis}

Descriptive statistics were used to summarize the basic characteristics of participants and all measurements. The $\chi^{2}$ test was used to evaluate the female/male ratio and acute/chronic ratio of participants. Mean differences between age groups were analyzed by Kruskal-Wallis analysis of variance. The effects of sex and age group were analyzed using the Wilcoxon rank-sum test for each variable (present pain, worst pain, depression score, and somatization score).

A $P$ value of $<0.05$ was considered to indicate statistical significance. SPSS for Windows version 12.0 (SPSS, Chicago, IL, USA) was used for all analyses.

\section{Results}

Descriptive data for the distribution of sex, muscle/TMJ pain ratio, and chronic pain, by age group, are shown in Table 1. The participants comprised 1,019 women and 418 men. The largest age group was Group B (25-34 years; including 215 women and $117 \mathrm{men}$ ), and the second largest age group was patients aged 55-64 years (205 women and 61 men). All age groups had significantly more women than men $\left(P<0.05, \chi^{2}\right.$ test $)$.

The ratio of participants with muscle pain (Ia or $\mathrm{Ib}$ ) to those with TMJ pain (IIIa or IIIb) was similar in all age groups. The proportion of patients with chronic illness (>6 months) was $38.4 \%, 35.5 \%, 38.4 \%, 35.7 \%, 30.8 \%$, and $21.3 \%$ in Groups A, B, C, D, E, and F, respectively. As compared with the other age groups, Group $\mathrm{F}$ had a significantly lower number of patients with chronic illness $\left(P<0.05, \chi^{2}\right.$ test $)$.

\section{Pain intensity}

Present pain intensity did not significantly differ by sex or age group (Fig. 1). Worst pain intensity did not significantly differ by sex, but age group had a significant effect $(P<0.001)$. Among women, worst pain intensity was significantly lower in Groups $\mathrm{E}$ and $\mathrm{F}$ than in Groups $\mathrm{B}$ and $\mathrm{C}(P<0.05)$ (Fig. 2). 


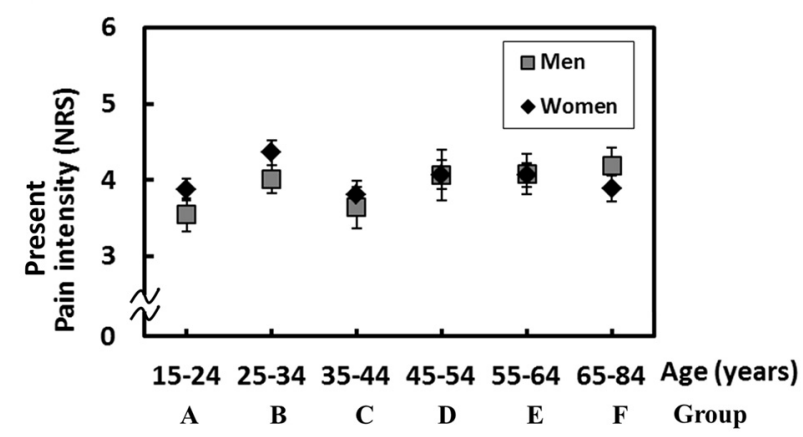

Fig. 1 Self-reported present pain intensity in each age group. There was no significant difference between sexes or among age groups. NRS, numeric rating scale.

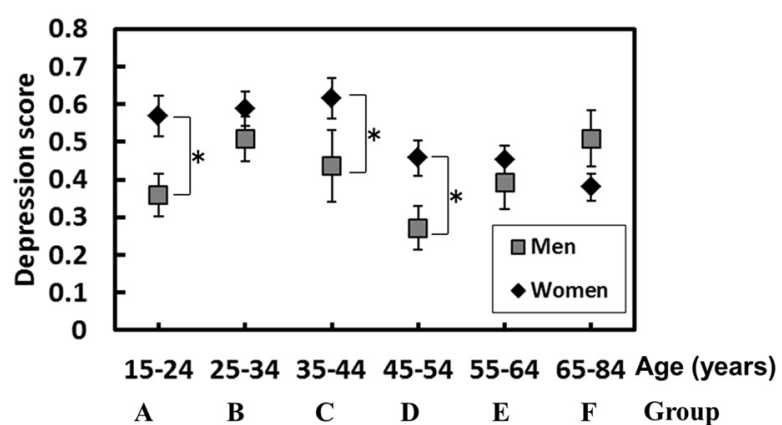

Fig. 3 Depression score in each age group. Depression scores were significantly higher for women in Groups A, C, and D than for men in the respective groups $(* P<0.05)$. Among women, depression score was significantly lower for Group $F$ than for Groups A through C.

\section{Psychosocial characteristics}

Depression scores were significantly higher among women in Groups A, C, and D than among men in the respective groups $(P<0.05)$. Age had a significant effect on depression score $(P<0.005)$. In same-sex comparisons, depression score was significantly lower for women in Group F than for those in Groups A, B, and C (Fig. 3).

Somatization score did not significantly vary by age group. Sex, by contrast, had a significant effect on somatization score $(P<0.001)$; in Groups A, B, C, and $\mathrm{D}$, somatization scores were significantly higher among women than among men $(P<0.05)$ (Fig. 4).

\section{Discussion}

This study enrolled 2.4 times as many women as men, which was similar to the ratio in a previous report (11). According to Levitt et al. (12), physical and psychological TMD symptoms are more severe among women than among men. This may partially explain the high female-to-male ratio among patients seeking treatment.

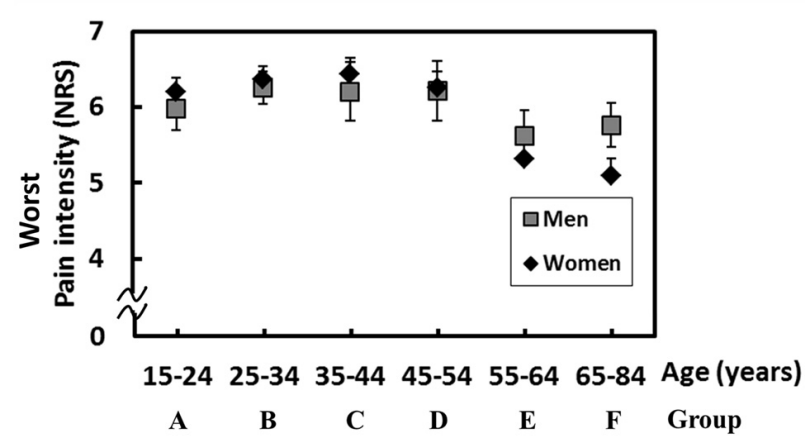

Fig. 2 Self-reported worst pain intensity during the past 6 months in each age group. There was no significant difference between sexes. Among women, worst pain intensity was significantly lower in Groups E and F than in Groups B and C $(P<0.05)$. NRS, numeric rating scale.

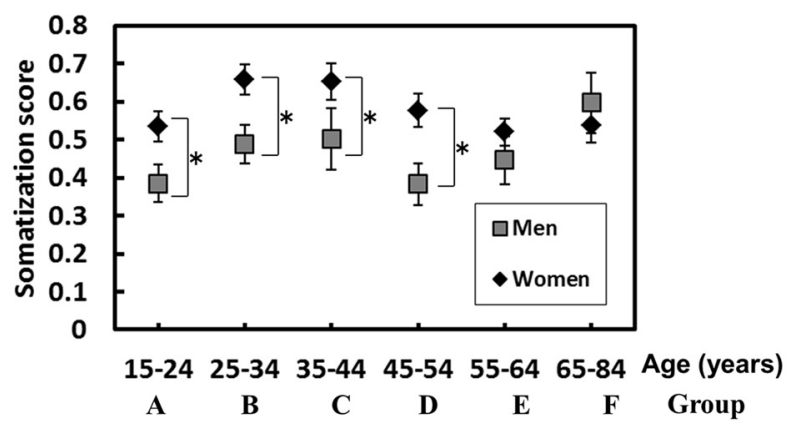

Fig. 4 Somatization score in each age group. There was no significant effect between sexes, but age had a significant effect $(P<0.001)$. In Groups A through D, scores were significantly higher for women than for men $\left({ }^{*} P<0.05\right)$.

Prior research revealed other factors that might contribute to such sex differences, including hormonal alterations $(13,14)$, blood pressure $(15)$, and psychological factors (16). Clinically, the main issues are whether the pain experience differs by sex and, if so, how this information can be used to improve treatment strategies and quality of life for those with pain (11).

Some studies have examined the relationship between TMD and age. Manfredini et al. identified at least two distinct age peaks within a patient population: one at about age 30 to 35 years, with disk displacement, and another at about age 50 to 55 years, with arthritis and/or arthrosis (17). Guarda-Nardini et al. identified two age peaks in TMD prevalence: one at around age 52 years for patients with joint crepitus from degenerative disease and another at around age 38 years for patients without joint crepitus (18). In the present study, the ratio of muscle to TMJ pain was very similar among age groups, but moredetailed examinations might detect differences in TMD types among age groups. Contrary to the popular view 
that TMD becomes more prevalent with age, most objective "signs" of TMD are either less frequent in the elderly or occur at approximately the same rate in all adult age groups. The prevalences of subjective complaints also decrease as the study population ages, and the demand for treatment declines accordingly (19).

In Group F, the number of patients with chronic illness ( $>6$ months) was lower than in the other age groups. Osterberg et al. found that the risk of craniomandibular dysfunction does not increase with age. However, awareness of such symptoms tends to decrease with age (2). Badel et al. classified patients as older ( $\geq 60$ years) and younger ( $<60$ years) and noted a high level of anxiety in both patient groups, regardless of the shorter duration of pain in older patients (20). Older patients may present earlier with TMD symptoms because they have more time to go to the hospital.

Many studies have examined the relationship between TMD and psychosocial factors. TMD symptoms are significantly associated with a number of general health and psychosomatic factors (21). Analysis of 5-year follow-up data from a population-based study indicated that depressive and anxiety symptoms were risk factors for TMD pain and that depressive symptoms are specific to joint pain, whereas anxiety symptoms are specific to muscle pain (22). Levitt et al. showed that psychological factors and stress do not progressively increase in severity or prevalence in older age groups. The overall severity of TMD symptoms did not increase in older groups (12), and the present study confirmed this finding.

Some studies examined sex differences in TMD in different age groups. Unell et al. reported that prevalences of TMD-related symptoms were higher in women than in men, but differences in an older group were smaller than those previously reported for younger individuals (23). The incidence of TMD in a cohort with depression was 2.65 times that of a cohort without depression, and the risk of developing TMD in relation to depression was higher in women than in men, which suggests that patients with depression have a higher risk of developing TMD (24). Giannakopoulos et al. found that depression may have an important role in chronic myofascial pain in women, whereas anxiety was not relevant for women or men assessed with the Hospital Anxiety and Depression Scale (25). Lajnert et al. found that patients reporting higher levels of depression were more inclined to somatization and had experienced more stress events in the past 6 months. They also reported that levels of depression, somatization, and anxiety are higher among patients with TMD than among healthy individuals (26).

Regarding worst pain intensity, values were signifi- cantly lower for women in Groups $\mathrm{E}$ and $\mathrm{F}$ than for those in Groups B and C in the present study. In addition, depression scores for women in Group $\mathrm{F}$ were significantly lower than for women in Groups A, B, and C. In older patients, psychosocial problems may be more evenly distributed between men and women. Some studies found that depression was more prevalent in elderly populations than in general populations $(27,28)$. However, in the present study, depression scores were significantly lower in elderly patients than in younger patients. Younger patients are more likely to have chronic TMD pain, which decreases with advancing age. This might explain why depression scores were higher among younger patients in this study.

In summary, the present study revealed some interesting features in a population of patients seeking TMD treatment at a specialized university clinic in Japan. Among women, worst pain intensity was lower for older TMD patients than for younger patients. Depressive symptoms were more prevalent among young women with TMD than among men, but this difference decreased and disappeared with age. Therefore, psychosocial problems and pain severity appear to decrease with age in TMD patients, particularly in women. Clinical treatment should thus be more conservative for older TMD patients.

\section{Acknowledgments}

This study was supported by a Grant-in-Aid for Scientific Research (C 26462959) from the Japanese Society for the Promotion of Science.

\section{References}

1. Carlsson GE (1999) Epidemiology and treatment need for temporomandibular disorders. J Orofac Pain 13, 232-237.

2. Osterberg T, Carlsson GE, Wedel A, Johansson U (1992) A cross-sectional and longitudinal study of craniomandibular dysfunction in an elderly population. J Craniomandib Disord 6, 237-245.

3. Salonen L, Helldén L, Carlsson GE (1990) Prevalence of signs and symptoms of dysfunction in the masticatory system: an epidemiologic study in an adult Swedish population. J Craniomandib Disord 4, 241-250.

4. Rutkiewicz T, Könönen M, Suominen-Taipale L, Nordblad A, Alanen P (2006) Occurrence of clinical signs of temporomandibular disorders in adult Finns. J Orofac Pain 20, 208-217.

5. De Boever JA, Carlsson GE, Klineberg IJ (2000) Need for occlusal therapy and prosthodontic treatment in the management of temporomandibular disorders. Part I. Occlusal interferences and occlusal adjustment. J Oral Rehabil 27, 367-379.

6. Dworkin SF, Von Korff M, LeResche L (1992) Epidemiologic studies of chronic pain: a dynamic-ecologic perspective. Ann 
Behav Med 14, 3-11.

7. Dworkin SF, Von Korff M, LeResche L (1990) Multiple pains and psychiatric disturbance: an epidemiologic investigation. Arch Gen Psychiatry 47, 239-244.

8. Dworkin SF, LeResche L (1992) Research diagnostic criteria for temporomandibular disorders: review, criteria, examinations and specifications, critique. J Craniomandib Disord 6, 301-355.

9. Blazer DG, Kessler RC, McGonagle KA, Swartz MS (1994) The prevalence and distribution of major depression in a national community sample: the National Comorbidity Survey. Am J Psychiatry 151, 979-986.

10. Müller F, Naharro M, Carlsson GE (2007) What are the prevalence and incidence of tooth loss in the adult and elderly population in Europe? Clin Oral Implants Res 18, Suppl 3, 2-14.

11. Dao TT, LeResche L (2000) Gender differences in pain. J Orofac Pain 14, 169-184.

12. Levitt SR, McKinney MW (1994) Validating the TMJ scale in a national sample of 10,000 patients: demographic and epidemiologic characteristics. J Orofac Pain 8, 25-35.

13. Fillingim RB, Maixner W, Girdler SS, Light KC, Harris MB, Sheps DS et al. (1997) Ischemic but not thermal pain sensitivity varies across the menstrual cycle. Psychosom Med 59, 512-520.

14. Pfleeger M, Straneva PA, Fillingim RB, Maixner W, Girdler SS (1997) Menstrual cycle, blood pressure and ischemic pain sensitivity in women: a preliminary investigation. Int $\mathrm{J}$ Psychophysiol 27, 161-166.

15. Fillingim RB, Maixner W (1996) The influence of resting blood pressure and gender on pain response. Psychosom Med 58, 326-332.

16. Fillingim RB, Keefe FJ, Light KC, Booker DK, Maixner W (1996) The influence of gender and psychological factors on pain perception. J Gender Cult Health 1, 21-36.

17. Manfredini D, Piccotti F, Ferronato G, Guarda-Nardini L (2010) Age peaks of different RDC/TMD diagnoses in a patient population. J Dent 38, 392-399.

18. Guarda-Nardini L, Piccotti F, Mogno G, Favero L, Manfredini
D (2012) Age-related differences in temporomandibular disorder diagnoses. Cranio 30, 103-109.

19. Greene CS (1994) Temporomandibular disorders in the geriatric population. J Prosthet Dent 72, 507-509.

20. Badel T, Simunković SK, Marotti M, Lovko SK, Kern J, Krolo I (2012) Study of temporomandibular joint disorder in older patients by magnetic resonance imaging. Gerodontology 29, e735-741.

21. Osterberg T, Carlsson GE (2007) Relationship between symptoms of temporomandibular disorders and dental status, general health and psychosomatic factors in two cohorts of 70-year-old subjects. Gerodontology 24, 129-135.

22. Kindler S, Samietz S, Houshmand M, Grabe HJ, Bernhardt O, Biffar R et al. (2012) Depressive and anxiety symptoms as risk factors for temporomandibular joint pain: a prospective cohort study in the general population. J Pain 13, 1188-1197.

23. Unell L, Johansson A, Ekbäck G, Ordell S, Carlsson GE (2012) Prevalence of troublesome symptoms related to temporomandibular disorders and awareness of bruxism in 65- and 75-year-old subjects. Gerodontology 29, e772-779.

24. Liao CH, Chang CS, Chang SN, Lane HY, Lyu SY, Morisky DE et al. (2011) The risk of temporomandibular disorder in patients with depression: a population-based cohort study. Community Dent Oral Epidemiol 39, 525-531.

25. Giannakopoulos NN, Keller L, Rammelsberg P, Kronmüller KT, Schmitter M (2010) Anxiety and depression in patients with chronic temporomandibular pain and in controls. J Dent 38, 369-376.

26. Lajnert V, Francisković T, Grzic R, Kovacević Pavicić D, Bakarbić D, Buković D et al. (2010) Depression, somatization and anxiety in female patients with temporomandibular disorders (TMD). Coll Antropol 34, 1415-1419.

27. Gatz M, Hurwicz ML (1990) Are old people more depressed? Cross-sectional data on Center for Epidemiological Studies Depression Scale factors. Psychol Aging 5, 284-290.

28. Djernes JK (2006) Prevalence and predictors of depression in populations of elderly: a review. Acta Psychiatr Scand 113, 372-387. 